\title{
Declaraciones nutricionales y de propiedades saludables aplicables a los productos de alimentación infantil en España y en la Unión Europea
}

Publicado en Internet:
9-enero-2014
Eduard Baladia:
info@grep-aedn.es Universidad de Alcalá de Henares, Madrid. España • `Plan integral de promoción de la salud mediante la

${ }^{a}$ Grupo de Revisión, Estudio y Posicionamiento de la Asociación Española de Dietistas-Nutricionistas (GREP-AEDN). España

- bescuela de Postgrado. Departamento de Ciencias de la Salud.

\author{
E. Baladiaa, J. Basulto ${ }^{b}$, M. Manera ${ }^{c}$
} actividad fisica y la alimentación saludable (PAAS). Generalitat de Cataluña. Barcelona. España.

que puede posicionar y diferenciar un producto o marca. El fraude en cuestiones en las que se requiere un análisis científico es difícilmente refutable por parte del consumidor. La legislación y los organismos científicos oficiales deben velar para que la comunicación sea veraz. El Reglamento 1924/2006 y la European Food Safety Authority (EFSA) tienen un papel fundamental, al ser el eje central sobre el que se desarrolla la creación y aceptación final de las declaraciones nutricionales y de propiedades saludables. Sin embargo, la coexistencia de legislación específica aplicable a los alimentos para lactantes y niños de corta edad y contemporánea al Reglamento 1924/2006, ha complicado la interpretación y aplicación del reglamento a dichos alimentos de especial consideración. Las Directivas 2006/141 y 2006/125 contienen apartados específicos de declaraciones nutricionales y de propiedades saludables no sometidas a aprobación por la EFSA y, en algunas ocasiones, el Reglamento 1924/2006 entra en conflicto con dichas directivas en esta materia. En esta revisión se desvelan algunas de las historias más accidentadas que envuelven el desarrollo de una legislación necesaria, ambiciosa y compleja.

\section{Nutrition and health claims on products for infant feeding in Spain and the European Union}

Nutrition and health claims are an added value that can position and differentiate a product or brand. Fraud issues in what scientific analysis is required are hardly refutable by the consumer. Legislation and official scientific bodies should ensure that the communication is truthful. Regulation 1924/2006 and the European Food Safety Authority (EFSA) have a key role, being the focus on the creation and final acceptance of nutrition and health claims. However, the coexistence of specific legislation applicable to foods for infants and young children to the Regulation 1924/2006, has complicated the interpretation

Key words: and application of the regulation to these foods of special consideration. Directives 2006/141 and $2006 / 125$ contain some specific sections on nutrition and health claims not subject to approval by the EFSA, and sometimes the Regulation 1924/2006 conflicts with these specific directives in this area. In this review, we reveal some of the roughest stories involving the development of a necessary, ambitious and complex legislation.

Cómo citar este artículo: Baladia E, Basulto J, Manera M. Declaraciones nutricionales y de propiedades saludables aplicables a los productos de alimentación infantil en España y en la Unión Europea. Rev Pediatr Aten Primaria. 2013;15:351-9. 


\section{INTRODUCCIÓN}

Las declaraciones nutricionales y de propiedades saludables no deben entenderse como una nueva tendencia publicitaria, de comunicación o "marketiniana", sino que vienen siendo, desde hace décadas, un valor añadido que puede posicionar y diferenciar a un producto o marca. Como prueba, los vestigios publicitarios en los periódicos pueden servir como repaso histórico del más que cuestionable reclamo publicitario poco controlado y fraudulento dirigido a la población general y también, cómo no, especialmente dedicado a la infancia (Figs. 1 y 2) ${ }^{1}$. Son declaraciones nutricionales todas aquellas comunicaciones que indiquen que un producto alimenticio contiene "un nutriente en especial”, y son declaraciones de propiedades saludables las comunicaciones que indiquen que un pro-

Figura 1. Anuncios publicados el 25 de agosto de 1903 en el periódico ABC-Madrid (página 11). En los tres anuncios se hace referencia a declaraciones de mejora de la salud, algunos de ellos son productos destinados a grupos de población especialmente vulnerables, los niños

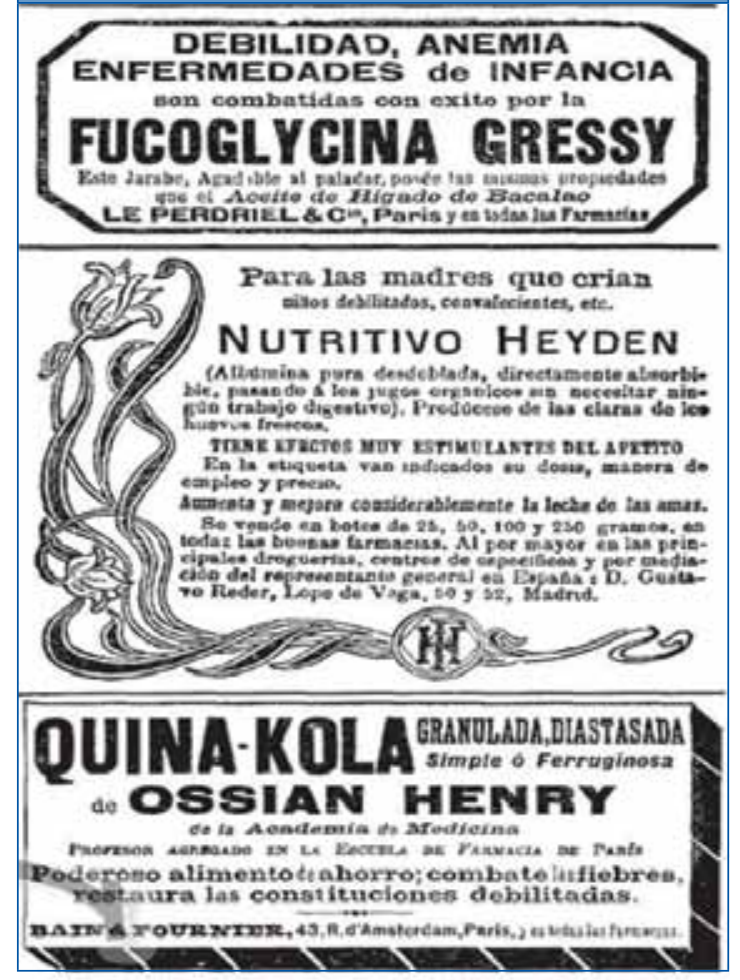

Figura 2. Anuncio publicado el 30 de agosto de 1951 en el periódico ABC-Madrid (página 4). Una conocida marca de bollería declara que su producto es "el alimento más nutritivo [declaración nutricional], sano y de fácil digestión [declaraciones de salud] para los niños"

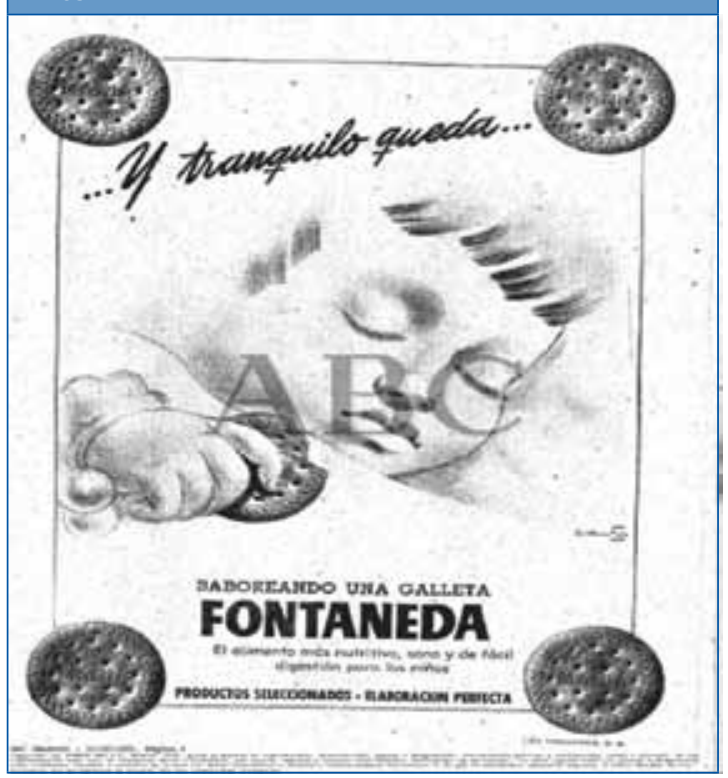

ducto alimenticio tiene además un "beneficio específico para la salud" (por su contenido en cierto nutriente, o bien porque ha demostrado, independientemente de su composición nutricional, afectar de forma beneficiosa a la salud).

El engaño o fraude en cuestiones tangibles, como por ejemplo la diferencia entre el aspecto de los alimentos en las fotografías de los envoltorios y el aspecto real de dichos alimentos cuando llegan al consumidor, es fácilmente identificable por parte de la mayoría de los consumidores. Sin embargo, el fraude en cuestiones menos tangibles, como por ejemplo la comunicación de pretendidas propiedades saludables, en la que es necesario un análisis del conocimiento científico, es difícilmente refutable para un consumidor medio, incluso para aquel que realmente está "normalmente informado"y es "razonablemente atento y perspicaz" ${ }^{1}$.

Si bien la Constitución Española reconoce el derecho a la salud, a la seguridad y a la veracidad en las informaciones (artículos 20, 43 y 51), es en las últimas décadas cuando se ha desarrollado en Europa y en España una legislación específica para proteger a los 
ciudadanos (y especialmente a los niños) ante los posibles riesgos derivados de la publicidad engañosa.

En este sentido, la Directiva 2006/141/CE, relativa a los preparados para lactantes y preparados de continuación ${ }^{2}$, constituye un avance al considerar explícitamente que "la publicidad de los preparados para lactantes se limitará a las publicaciones especializadas en la asistencia infantil y a las publicaciones científicas", y detallando que "contendrán únicamente información de carácter científico y objetivo". Asimismo, dispone que la información "no deberá insinuar ni hacer creer que la alimentación con biberón es equivalente o superior a la lactancia materna". En cambio, la Directiva 2006/125/ $\mathrm{CE}^{3}$, relativa a los alimentos elaborados a base de cereales y alimentos infantiles para lactantes y niños de corta edad, no incorpora (para sorpresa de los autores) ninguna consideración con respecto a la publicidad de dichos alimentos.

Sin embargo, se trata de un tema mucho más complejo de lo que puede parecer a simple vista, ya que, además de dichos actos legislativos que conforman el marco general de protección al consumidor, el código de autorregulación de la publicidad de alimentos dirigida a menores para la prevención de la obesidad y salud (código PAOS) ${ }^{4}$, desarrollado con el concurso de la Administración y las organizaciones de consumidores, ha ayudado (en teoría) a establecer un acuerdo voluntario de mínimos para una publicidad menos engañosa dirigida la población infantil. Esta clase de convenios son, para algunos expertos en salud pública, tan ineficaces y contraproducentes como los firmados por las industrias del tabaco y del alcohol ${ }^{5}$. Una revisión sistemática de la bibliografía científica publicada en julio de 2013 observó que los códigos voluntarios de regulación de la publicidad dirigida a niños no garantizan la disminución de la propaganda de alimentos que "socavan las dietas saludables"6.

Sea como fuere, en 2006 apareció un reglamento ambicioso: el Reglamento $\mathbf{1 9 2 4 / 2 0 0 6}$, relativo a las declaraciones nutricionales y de propiedades saludables en los alimentos ${ }^{7}$, que constituyó el primer acto legislativo dirigido específicamente a establecer "las reglas básicas" para la creación y utili- zación de las declaraciones nutricionales y de propiedades saludables aplicables a los alimentos, a nivel europeo. No obstante, como se detalla más adelante, resultará ser una legislación bastante más compleja de aplicar de lo que se podría esperar, y que en el caso de alimentos destinados a la población infantil, debe aplicarse de acuerdo a otras directivas.

A continuación se realiza un breve repaso en relación a la legislación aplicable en materia de declaraciones nutricionales y de propiedades saludables.

\section{REGLAMENTO 1924/2006 DE DECLARACIONES NUTRICIONALES Y DE PROPIEDADES SALUDABLES: REGULACIÓN ACCIDENTADA}

El Reglamento 1924/2006, relativo a las declaraciones nutricionales y de propiedades saludables en los alimentos pretendía establecer, por primera vez, "las reglas del juego" para regular las crecientes declaraciones nutricionales y alegaciones de salud atribuidas a los alimentos. Por un lado, definió los principios generales que deben cumplir todas las declaraciones, así como las condiciones específicas para el uso de tales declaraciones. Por otro lado, estableció una lista positiva (y por lo tanto limitada) de declaraciones nutricionales, específica y exhaustivamente descritas en su anexo. Finalmente, sentó además las bases para que las empresas $u$ organismos interesados enviaran peticiones de autorización de declaraciones de propiedades saludables. Sin embargo, no fue hasta 2008 (dos años después) cuando la Comisión desveló el importante significado de "pruebas científicas generalmente aceptadas" al publicar el Reglamento (CE) n. ${ }^{0} 353 / 2008^{8}$, en el que se concreta que a nivel documental:

1. Se haga un "examen exhaustivo y sistemático de datos procedentes de seres humanos, incluyendo todos los datos y sopesándolos".

2. Se incorporen datos tanto publicados como no publicados, así como datos positivos (a favor de 
la declaración) y datos negativos (en contra de la declaración).

3. Y se evalúen los siguientes tipos de estudios (están ordenados de mayor a menor grado de evidencia): a) estudios de intervención en humanos; b) estudios observacionales en humanos, c) estudios mecanísticos en humanos; d) estudios con modelos animales, y e) estudios con modelos celulares.

Tras varias modificaciones del Reglamento 1924/2006, en 2012 quedó constituida la última lista que incluye las declaraciones nutricionales que se pueden realizar. En la Tabla 1 (parte 1) se detalla la lista de declaraciones nutricionales permitidas hasta la fecha.

Paralelamente, la European Food Safety Authority (EFSA), a marchas forzadas, publicó opiniones de todas las solicitudes de declaraciones de salud realizadas por los operadores comerciales (empresas).

Finalmente, el 16 de mayo de 2012, la Comisión publica la lista de declaraciones autorizadas de propiedades saludables de los alimentos distintas de las relativas a la reducción del riesgo de enfermedad y al desarrollo y la salud de los niños ${ }^{9}$. Hasta tres modificaciones se realizan de dicho documento en menos de cinco meses ${ }^{10-12}$.

El 24 de enero de 2013, se publicaron las directrices específicas para asegurar que se cumplirían las condiciones específicas de declaraciones de sa$\operatorname{lud}^{13}$. La Comisión lo dejó claro: las únicas declaraciones de salud que pueden utilizarse son las autorizadas. Así, si un agente de una empresa alimentaria ("food business operator" en palabras de la EFSA) desea utilizar una declaración de salud, debe acudir, hoy por hoy, al portal web http:// ec.europa.eu/nuhclaims/?event=search\&status_ ref_id $=4$

El 12 de junio de 2013, se publicó la que quizás fue la penúltima página de la accidentada historia del Reglamento 1924/2006: "la corrección de errores de la corrección de errores"14.

Sin embargo, cabe destacar que existe aún una tarea pendiente por parte de la Comisión, el esta-

Tabla 1. Declaraciones nutricionales permitidas hasta la fecha (noviembre de 2013) por el Reglamento 1924/2006 y aplicables a priori a los alimentos para lactantes y para niños de corta edad, excepto si entran en conflicto con las Directivas 2006/141 y 2006/125 (parte 1). Declaraciones nutricionales permitidas por la Directiva 2006/141 (parte 2)

\section{Parte 1. Declaraciones nutricionales (anexo Reglamento 1924/2006)}
1. Bajo valor energético
16. Bajo contenido en sodio/sal
2. Valor energético reducido
17. Muy bajo contenido en sodio/sal
3. Sin aporte energético
18. Sin sodio/sin sal
4. Bajo contenido en grasa
19. Sin sodio/sin sal añadidos
5. Sin grasa
6. Bajo contenido en grasas saturadas
20. Fuente de fibra
21. Alto contenido en fibra
7. Sin grasas saturadas
22. Fuente de proteínas
8. Fuente de ácidos grasos $\omega^{-3}$
9. Alto contenido en ácidos grasos $\omega-3$
23. Alto contenido en proteínas
24. Fuente de [vitaminas/minerales]
25. Alto contenido de [vitaminas/minerales]
10. Alto contenido en grasas monoinsaturadas
26. Contiene [nutriente u otras sustancias]
12. Alto contenido en grasas insaturadas
13. Bajo contenido en azúcares
14. Sin azúcares
15. Sin azúcares añadidos
27. Mayor contenido de [nutriente]
28. Contenido reducido de [nutriente]
29. Light/lite (ligero)
30. Naturalmente/Natural

Parte 2. Declaraciones nutricionales (Directiva 2006/141)

1. Únicamente lactosa: la lactosa será el único carbohidrato presente

2. Ausencia de lactosa: el contenido en lactosa no superará $2,5 \mathrm{mg} / 100 \mathrm{~kJ}(10 \mathrm{mg} / 100 \mathrm{kcal})$

3. Ácidos grasos poliinsaturados de cadena larga añadidos o una alegación nutricional equivalente relacionada con la adición de ácido docosahexanoico: el contenido en ácido docosahexanoico no será inferior a un 0,2\% del contenido total en ácidos grasos

4. Adición de taurina, fructooligosacáridos y galactooligosacáridos, nucleótidos: añadidos voluntariamente en una medida apropiada para el uso particular previsto por parte de los lactantes y de conformidad con las condiciones establecidas en el anexo I 
blecimiento de los "perfiles nutricionales" de los que habla el artículo 4 del Reglamento, y que deberían haber sido aprobados a más tardar el 19 de enero de 2009. Pese a que no han sido aún aprobados, existe una propuesta de la EFSA publicada el 25 de febrero de $2008^{15}$, y llevada ante la Comisión Europea con fecha 13 de febrero de $2009^{16}$. Su publicación asegurará que alimentos que sobrepasen ciertos valores en cuanto a sodio, grasas saturadas y azúcares (nutrientes considerados como "de interés para la salud pública"17 al relacionanarse de forma directa con las principales enfermedades crónicas de nuestra sociedad) no puedan contener ninguna declaración de propiedad saludable.

\section{DECLARACIONES NUTRICIONALES Y DE PROPIEDADES SALUDABLES EN PRODUCTOS DE ALIMENTACIÓN INFANTIL}

Si bien el Reglamento 1924/2006 ${ }^{18}$ debe aplicarse a la comunicación de declaraciones nutricionales y de propiedades saludables de todos los alimentos, en el artículo 1 punto 5 se dice expresamente que dicho Reglamento se aplicará sin perjuicio de la $\mathrm{Di}$ rectiva 89/398/CEE (algo que hoy por hoy no tiene mucho sentido, ya que se trata de una directiva derogada por la Directiva 2009/39/CE ${ }^{19}$. El Reglamento 1924/2006 debe aplicarse de forma paralela o teniendo en cuenta:

- Directiva 2006/141/CE, de 22 de diciembre de 2006, relativa a los preparados para lactantes y preparados de continuación ${ }^{2}$.

- Directiva 2006/125/CE, de 5 de diciembre de 2006, relativa a los alimentos elaborados a base de cereales y alimentos infantiles para lactantes y niños de corta edad ${ }^{3}$.

Hasta aquí podría pensarse que está todo bastante claro: antes de emitir una declaración de salud relacionada con un alimento dirigido a la infancia, bastaría con revisar el Reglamento 1924/2006 y las dos directivas recién citadas. Sin embargo, en relación a las declaraciones nutricionales, cuando se analizan en profundidad el Reglamento 1924/2006 y las Directivas específicas 2006/141 y 2006/1252,3, se encuentran varias incoherencias y dificultades para entender hasta qué punto hay que utilizar la información del Reglamento 1924/2006 o bien las directivas específicas de 2006.

Las directivas específicas de $2006^{2,3}$, establecen en sus anexos la cantidad mínima y máxima de todos los nutrientes que pueden contener los alimentos destinados a lactantes (menos de 12 meses de edad) y niños de corta edad (entre uno y tres años) dejando, en consecuencia, poco espacio de diferenciación entre productos, y haciendo poco útil (al menos para las empresas) el Reglamento 1924/2006. Asimismo, en el anexo IV de la Directiva $2006 / 141^{2}$, existe un apartado específico de declaraciones nutricionales y de declaraciones de propiedades saludables no aprobadas por la EFSA. Según dicha directiva, se permiten las declaraciones nutricionales ofrecidas en la Tabla 1 (parte 2$)^{2}$. Si bien dichas declaraciones nutricionales entran en conflicto con el Reglamento 1924/2009, entendemos que el reglamento específico prevalece por encima del general.

En esta misma línea, en los anexos de las directivas específicas de $2006^{2,3}$, se establecen valores de referencia para el etiquetado de propiedades nutricionales de alimentos destinados a lactantes y niños de corta edad, que entran en conflicto con los valores definidos por el Reglamento (UE) n.o $1169 / 2011$ adecuados para adultos ${ }^{20}$ (este reglamento derogó la Directiva 90/496/CEE ${ }^{21}$, modificación que aún no se ha hecho efectiva en el Reglamento 1924/2006). Se puede entender que deben usarse los valores de referencia de las directivas específicas de $2006^{2,3}$, adaptados a la población diana de las declaraciones nutricionales, aunque esta es una aseveración no resuelta en la legislación vigente.

En relación a las declaraciones de propiedades saludables, el Reglamento 1924/2006 ${ }^{18}$ establece que su solicitud debe hacerse con arreglo al artículo 14 "Declaraciones de reducción del riesgo de enfermedad y declaraciones relativas al desarrollo y la salud de los niños". De entre las declaraciones de propiedades saludables relacionadas con el de- 
sarrollo y salud de los niños, solamente 11 declaraciones han sido autorizadas por la EFSA (aunque aún no aprobadas por la Comisión) (Tabla 2, parte 1). Sin embargo, en el anexo IV de la directiva específica 2006/1412 existe una declaración más de propiedades saludables que no ha sido sometida a la opinión de la EFSA (Tabla 2, parte 2). De nuevo, la directiva específica entra en conflicto con el Regalmento 1924/2006.

En este sentido, tal y como se ha comentado anteriormente, en el caso de las declaraciones de propiedades saludables establecidas mediante el procedimiento del Reglamento 1924/2006 y que establecen la condición de poderse hacer, si se cumple la declaración nutricional "ser fuente de [nutriente]", no queda muy claro si deberían consultarse los datos de valores de referencia para el etiquetado de propiedades nutricionales de alimentos del Reglamento (UE) n.ㅇ 1169/201120 (para adultos) o bien de las Directivas 2006/141 y 2006/125 2,3 adecuadas para la población infantil.

Para terminar con dicha indefinición (o eso imaginan los autores del presente artículo), el 12 de junio de 2013 se publicó el "nuevo" Reglamento (UE) n. $.609 / 2013$ relativo a los alimentos destinados a los lactantes y niños de corta edad ${ }^{22}$, que derogará las Directivas específicas 2006/141 y 2006/125.

Este reglamento mantiene el fomento de la lactancia materna en todas las comunicaciones comerciales de productos destinados a población infantil, e indica que a más tardar el 20 de julio de 2015 se redactarán actos delegados de requisitos específicos sobre la autorización de declaraciones nutricionales y de propiedades saludables relativos a los productos para lactantes y niños de corta edad.

\section{CONCLUSIONES}

Más allá de lo que es o no legal en estos momentos de transición (hasta 2015) en materia de declaraciones nutricionales o de propiedades saludables aplicables a los alimentos infantiles, a modo de entender de los autores del presente artículo, partidarios del principio de precaución y que preten- den ser más severos que la EFSA ${ }^{23}$, los profesionales sanitarios que actualmente deseen establecer o comprobar las declaraciones nutricionales y de propiedades saludables aplicables a un alimento destinados a lactantes y niños de corta edad deberían:

- Asegurarse de que se cumple con el fomento de la protección de la lactancia materna en todas las comunicaciones del producto (Reglamento [UE] n.․ 609/2013).

- Declaraciones nutricionales:

- Comprobar que el producto cumple con las declaraciones nutricionales admitidas en el anexo 1 del Reglamento 1924/2006, consultando (tal y como marca el reglamento) los valores de referencia para el etiquetado de propiedades nutricionales para adultos (Reglamento [UE] n.o 1169/2011).

- Comprobar si cumple, además, con las declaraciones nutricionales admitidas en el anexo 1 del Reglamento 1924/2006, consultando (tal y como sería lógico hacer) los valores de referencia para el etiquetado de propiedades nutricionales de alimentos destinados a lactantes y niños de corta edad (Directivas 2006/141 y 2006/125).

- Comprobar si dichas declaraciones nutricionales están recogidas en el anexo IV de la directiva 2006/141.

- Declaraciones de propiedades saludables:

- Comprobar las declaraciones de propiedades saludables aprobadas por la EFSA hasta la fecha (Tabla 2, parte 1). Deberían cumplirse las condiciones establecidas, en principio consultando (tal y como marca el reglamento) los valores de referencia para el etiquetado de propiedades nutricionales para adultos (Reglamento [UE] n.․ 1169/2011), pero recomendamos además constatar si se cumplen, consultando (tal y como sería lógico hacer) los valores de referencia para el etiquetado de propiedades nutricionales de alimentos destinados a lactantes y niños de corta edad (Directivas 2006/141 y 2006/125). 


\begin{tabular}{|c|c|c|c|c|}
\hline \multicolumn{5}{|c|}{ Parte 1. Declaraciones de propiedades saludables Reglamento 1924/2006 } \\
\hline Nutriente & & Declaración & Condición & $\begin{array}{l}\text { Opinión } \\
\text { de la EFSA }\end{array}$ \\
\hline \multirow[t]{3}{*}{$\begin{array}{l}\text { Ácido } \\
\text { docosahexaenoico } \\
\text { (DHA) }\end{array}$} & \multicolumn{2}{|c|}{$\begin{array}{l}\text { La ingesta maternal de DHA maternal } \\
\text { contribuye al desarrollo normal del } \\
\text { cerebro del feto y los recién nacidos } \\
\text { alimentados con leche materna }\end{array}$} & \multirow{2}{*}{$\begin{array}{l}\text { La afirmación solo puede utilizarse para } \\
\text { alimentos que proporcionen una ingesta } \\
\text { diaria de al menos } 200 \mathrm{mg} \text { de DHA }\end{array}$} & O-2008-773 \\
\hline & \multicolumn{2}{|c|}{$\begin{array}{l}\text { La ingesta maternal de DHA contribuye } \\
\text { al desarrollo normal del ojo del feto y } \\
\text { de los lactantes alimentados con leche } \\
\text { materna }\end{array}$} & & $\begin{array}{l}\text { O-2008-211 } \\
\text { O-2008-688 } \\
\text { O-2008-689 }\end{array}$ \\
\hline & \multicolumn{2}{|c|}{$\begin{array}{l}\text { La ingesta de DHA contribuye al } \\
\text { desarrollo visual normal de los lactantes } \\
\text { hasta } 12 \text { meses de edad }\end{array}$} & $\begin{array}{l}\text { Los alimentos deben contener al menos el } \\
0,3 \% \text { del total de ácidos grasos en forma de } \\
\text { DHA }\end{array}$ & Q-2008-675 \\
\hline $\begin{array}{l}\text { Ácidos grasos } \\
\text { esenciales } \\
\alpha \text {-linolénico (ALA) } \\
\text { y linoleico (LA) }\end{array}$ & \multicolumn{2}{|c|}{$\begin{array}{l}\text { Se necesitan los ácidos grasos esenciales } \\
\text { para el crecimiento normal y el desarrollo } \\
\text { de los niños }\end{array}$} & $\begin{array}{l}\text { El efecto beneficioso se obtiene con una } \\
\text { ingesta diaria de } 2 \mathrm{~g} \text { de ácido } Đ \text {-linolénico } \\
\text { (ALA) y una ingesta diaria de } 10 \mathrm{~g} \text { de ácido } \\
\text { linoleico (LA) }\end{array}$ & Q-2008-079 \\
\hline Calcio y vitamina $\mathrm{D}$ & \multicolumn{2}{|c|}{$\begin{array}{l}\text { El calcio y la vitamina D son necesarios } \\
\text { para el crecimiento y desarrollo normal } \\
\text { de los huesos en los niños }\end{array}$} & \multirow{7}{*}{$\begin{array}{l}\text { Puede realizarse la declaración cuando el } \\
\text { alimento es, por lo menos, fuente de dicho } \\
\text { nutriente según se define en el anexo del } \\
\text { Reglamento 1924/2006 }\end{array}$} & Q-2008-116 \\
\hline Calcio & \multicolumn{2}{|c|}{$\begin{array}{l}\text { El calcio es necesario para el crecimiento } \\
\text { normal y el desarrollo de los huesos en } \\
\text { los niños }\end{array}$} & & Q-2008-322 \\
\hline Vitamina D & \multicolumn{2}{|c|}{$\begin{array}{l}\text { La vitamina D es necesaria para el } \\
\text { crecimiento normal y el desarrollo de los } \\
\text { huesos en los niños }\end{array}$} & & Q-2008-323 \\
\hline Fósforo & \multicolumn{2}{|c|}{$\begin{array}{l}\text { El fósforo es necesario para el } \\
\text { crecimiento normal y el desarrollo de los } \\
\text { huesos en los niños }\end{array}$} & & O-2008-217 \\
\hline Yodo & \multicolumn{2}{|c|}{$\begin{array}{l}\text { El yodo contribuye al crecimiento normal } \\
\text { de los niños }\end{array}$} & & Q-2008-324 \\
\hline Hierro & \multicolumn{2}{|c|}{$\begin{array}{l}\text { El hierro contribuye al desarrollo } \\
\text { cognitivo normal de los niños }\end{array}$} & & Q-2008-325 \\
\hline Proteína & \multicolumn{2}{|c|}{$\begin{array}{l}\text { Las proteínas son necesarias para el } \\
\text { crecimiento y desarrollo normal de los } \\
\text { huesos en los niños }\end{array}$} & & Q-2008-326 \\
\hline \multicolumn{5}{|c|}{ Parte 2: Declaraciones de propiedades saludables Directiva 2006/141 } \\
\hline \multicolumn{2}{|c|}{ Declaración } & \multicolumn{3}{|c|}{ Condición } \\
\hline \multicolumn{2}{|c|}{$\begin{array}{l}\text { Reducción del riesgo de alergia } \\
\text { a las proteínas de la leche. } \\
\text { Esta alegación de propiedades } \\
\text { saludables puede ir acompañada } \\
\text { de términos que hagan referencia } \\
\text { a una propiedad alergénica } \\
\text { reducida o antigénica reducida }\end{array}$} & \multicolumn{3}{|c|}{$\begin{array}{l}\text { (a) debe disponerse de datos objetivos y verificados científicamente como prueba de las } \\
\text { propiedades alegadas } \\
\text { (b) los preparados para lactantes cumplirán lo establecido en el punto } 2.2 \text { del anexo l y la } \\
\text { cantidad de proteína inmunorreactiva medida con métodos generalmente aceptados es } \\
\text { inferior al } 1 \% \text { de las sustancias nitrogenadas del preparado } \\
\text { (c) se indica en la etiqueta que el producto no debe ser consumido por lactantes alérgicos a } \\
\text { las proteínas intactas de que procede, salvo que se compruebe mediante ensayos clínicos } \\
\text { generalmente aceptados que el preparado para lactantes es tolerado por más del } 90 \% \text { de } \\
\text { lactantes (intervalo de confianza del } 95 \% \text { ) hipersensibles a las proteínas de que procede } \\
\text { el hidrolizado } \\
\text { (d) los preparados para lactantes administrados oralmente no deben inducir sensibilización } \\
\text { en animales frente a las proteínas intactas de que procede el preparado para lactantes }\end{array}$} \\
\hline
\end{tabular}


- Comprobar la declaración de propiedad saludable aceptada en el anexo IV de la directiva 2006/141.

\section{CONFLICTO DE INTERESES}

Los autores declaran no presentar conflictos de intereses en relación con la preparación y publicación de este artículo.

\section{ABREVIATURAS}

EFSA: European Food Safety Authority.

\section{BIBLIOGRAFİA}

1. Baladia E. Módulo: Declaraciones Nutricionales y de Propiedades Saludables del Máster Nutrición Comunitaria y Nutrición Clínica. Alicante: Universidad de Alicante; 2013 [en línea] [consultado el 22/11/2013]. Disponible en http://rua.ua.es/dspace/bitstream/10045/28217/1/Modulo_Reglamento_Universidad_de_Alicante_2013_3-1_copia_2.pdf

2. Directiva 2006/141/CE de la Comisión, de 22 de diciembre de 2006, relativa a los preparados para lactantes y preparados de continuación y por la que se modifica la Directiva 1999/21/CE. Diario Oficial de la Unión Europea, núm. 401 de 30 de diciembre de 2006. p. 1-33.

3. Directiva 2006/125/CE de la Comisión, de 5 de diciembre de 2006, relativa a los alimentos elaborados a base de cereales y alimentos infantiles para lactantes y niños de corta edad (Versión codificada). Diario Oficial de la Unión Europea, núm. 339 de 6 de diciembre de 2006. p. 16-35.

4. Ministerio de Sanidad, Servicios Sociales e Igualdad (MSSSI), Federación Española de Industrias de la Alimentación y Bebidas (FIAB), Autocontrol, Asociación Nacional de Gramdes Empresas de Distribución (ANGED), Asociación Española de Distribuidores, Autoservicios y Supermercadas (ASEDAS), Asociación de Cadenas Españolas de Supermercados (ACES), et al. Código de autorregulación de la publicidad de alimentos dirigida a menores, prevención de la obesidad y salud (código PAOS). Madrid: MSSSI-FIAB-Autocontrol-ANGED-ASEDAS-ACES-FEHR-FEHRCAREM; 2012 [en línea] [consultado el 13/01/2013]. Disponible en www.naos.aesan.msssi.gob.es/naos/ficheros/ empresas/CODIGO_PAOS_2012.pdf
5. Royo-Bordonada MA, Martínez-Huedo MA. Evaluation of compliance with the self-regulation agreement of the food and drink vending machine sector in primary schools in Madrid, Spain, in 2008. Gac Sanit SESPAS. 2013 Jul 9 [en línea]. Disponible en www.elsevier.es/eop/S0213-9111(13)00119-2.pdf

6. Galbraith-Emami S, Lobstein T. The impact of initiatives to limit the advertising of food and beverage products to children: a systematic review. Obes Rev Off J Int Assoc Study Obes. 2013. [En prensa]

7. Reglamento (CE) n. ${ }^{\circ}$ 1924/2006 del Parlamento Europeo y del Consejo, de 20 de diciembre de 2006, relativo a las declaraciones nutricionales y de propiedades saludables en los alimentos. Diario Oficial de la Unión Europea, núm. 404 de 30 de diciembre de 2006. p. 9-25

8. Reglamento (CE) n. ${ }^{\circ}$ 353/2008 de la Comisión, de 18 de abril de 2008, por el que se establecen normas de desarrollo para las solicitudes de autorización de declaraciones de propiedades saludables con arreglo al artículo 15 del Reglamento (CE) n. ${ }^{\circ}$ 1924/2006 del Parlamento Europeo y del Consejo. Diario Oficial de la Unión Europea, núm. 109 de 19 de abril de 2008. p. 11-6.

9. Reglamento (UE) n. ${ }^{\circ}$ 432/2012 de la Comisión, de 16 de mayo de 2012, por el que se establece una lista de declaraciones autorizadas de propiedades saludables de los alimentos distintas de las relativas a la reducción del riesgo de enfermedad y al desarrollo y la salud de los niños. Diario Oficial de la Unión Europea, núm. 136 de 25 de mayo de 2012. p. 1-40.

10. Reglamento (UE) n. ${ }^{\circ}$ 536/2013 de la Comisión, de 11 de junio de 2013, que modifica el Reglamento (UE) n. ${ }^{\circ} 432 / 2012$, por el que se establece una lista de declaraciones autorizadas de propiedades saludables de los alimentos distintas de las relativas a la reduc- 
ción del riesgo de enfermedad y al desarrollo y la salud de los niños. Diario Oficial de la Unión Europea (DOUE), núm. 160, de 12 de junio de 2013. p. 4-8.

11. Reglamento (UE) n. ${ }^{\circ} 851 / 2013$ de la Comisión, de 3 de septiembre de 2013, por el que se autorizan determinadas declaraciones de propiedades saludables en los alimentos, distintas de las relativas a la reducción del riesgo de enfermedad y al desarrollo y la salud de los niños, y se modifica el Reglamento (UE) n. ${ }^{\circ}$ 432/2012. Diario Oficial de la Unión Europea (DOUE), núm. 235, de 4 de septiembre de 2013. p. 3-7.

12. Reglamento (UE) n. ${ }^{\circ} 1018 / 2013$ de la Comisión, de 23 de octubre de 2013, que modifica el Reglamento (UE) n. ${ }^{\circ} 432 / 2012$, por el que se establece una lista de declaraciones autorizadas de propiedades saludables de los alimentos distintas de las relativas a la reducción del riesgo de enfermedad y al desarrollo y la salud de los niños. Diario Oficial de la Unión Europea (DOUE), núm. 282, de 24 de octubre de 2013. p. 43-5.

13. Decisión de Ejecución de la Comisión, de 24 de enero de 2013, por la que se adoptan directrices para la aplicación de las condiciones específicas relativas a las declaraciones de propiedades saludables establecidas en el artículo 10 del Reglamento (CE) n. ${ }^{\circ}$ 1924/2006 del Parlamento Europeo y del Consejo. Diario Oficial de la Unión Europea, núm. 22 de 25 de enero de 2013. p. 25-8.

14. Corrección de errores de la corrección de errores del Reglamento (CE) n. ${ }^{\circ}$ 1924/2006 del Parlamento Europeo y del Consejo, de 20 de diciembre de 2006, relativo a las declaraciones nutricionales y de propiedades saludables en los alimentos. Diario Oficial de la Unión Europea (DOUE), núm. 160, de 12 de junio de 2013. p. 15.

15. EFSA Panel on Dietetic Products, Nutrition and Allergies (NDA). he setting of nutrient profiles for foods bearing nutrition and health claims pursuant to $\mathrm{Ar}$ ticle 4 of the Regulation (EC) No 1924/2006-Scientific Opinion of the Panel on Dietetic Products, Nutrition and Allergies. The EFSA J. 2008;644:1-44.

16. European Commission. Working document on the setting of nutrient profiles. Brussels; 2009.

17. Salas-Salvadó J, Martín Bermudo F, Martínez de Victoria E, Picó Segura C, Vidal Caru M, Gil Hernández A, et al. Informe del Comité Científico de la Agencia Española de Seguridad Alimentaria y Nutrición (AESAN) sobre criterios para incentivar la disminución del contenido de determinados nutrientes en los alimentos transformados, cuya reducción es de interés para la salud pública. Rev Comité Científico AESAN. 2013;15:43-56.

18. Corrección de errores del Reglamento (CE) n. ${ }^{\circ}$ 1924/2006 del Parlamento Europeo y del Consejo, de 20 de diciembre de 2006 , relativo a las declaraciones nutricionales y de propiedades saludables en los alimentos. Diario Oficial de la Unión Europea, núm. 12 de 18 de enero de 2007, págs. 3-18.

19. Directiva 2009/39/CE del Parlamento Europeo y del Consejo, de 6 de mayo de 2009, relativa a los productos alimenticios destinados a una alimentación especial (versión refundida). Diario Oficial de la Unión Europea (DOUE), núm. 124, de 20 de mayo de 2009. p. 21-9.

20. Reglamento (UE) n. ${ }^{\circ} 1169 / 2011$ del Parlamento Europeo y del Consejo, de 25 de octubre de 2011, sobre la información alimentaria facilitada al consumidor y por el que se modifican los Reglamentos (CE) n. ${ }^{\circ}$ 1924/2006 y (CE) n. ${ }^{\circ}$ 1925/2006 del Parlamento Europeo y del Consejo, y por el que se derogan la Directiva 87/250/CEE de la Comisión, la Directiva 90/496/CEE del Consejo, la Directiva 1999/10/CE de la Comisión, la Directiva 2000/13/CE del Parlamento Europeo y del Consejo, las Directivas 2002/67/CE, y 2008/5/CE de la Comisión, y el Reglamento (CE) n. ${ }^{\circ}$ 608/2004 de la Comisión. Diario Oficial de la Unión Europea (DOUE), núm. 304, de 22 de noviembre de 2011, p. 18-63.

21. Directiva del Consejo, de 24 de septiembre de 1990, relativa al etiquetado propiedades nutritivas de productos alimenticios. Diario Oficial de las Comunidades Europeas, núm. 276 de 6 de octubre de 1990. p. 40-4.

22. Reglamento (UE) n. ${ }^{\circ} 609 / 2013$ del Parlamento Europeo y del Consejo, de 12 de junio de 2013, relativo a los alimentos destinados a los lactantes y niños de corta edad, los alimentos para usos médicos especiales y los sustitutivos de la dieta completa para el control de peso y por el que se derogan la Directiva 92/52/CEE del Consejo, las Directivas 96/8/CE, 1999/21/CE, 2006/125/CE y 2006/141/CE de la Comisión, la Directiva 2009/39/CE del Parlamento Europeo y del Consejo y los Reglamentos (CE) n. ${ }^{\circ}$ 41/2009 y (CE) n. ${ }^{\circ}$ 953/2009 de la Comisión. Diario Oficial de la Unión Europea (DOUE), núm. 181, de 29 de junio de 2013. p. 35-56.

23. Baladia E, Basulto J, Manera M. Health claims. More severe than the European Food Safety Authority (EFSA): to be or not be? Ann Nutr Metab. 2013; 63(suppl 1):150. 